\title{
Samuel Beckett, Les Années Godot. Lettres II 1941-1956
}

\section{Stefano Genetti}

\section{(Q) OpenEdition}

\section{Journals}

\section{Edizione digitale}

URL: http://journals.openedition.org/studifrancesi/5427

DOI: 10.4000/studifrancesi.5427

ISSN: 2427-5856

\section{Editore}

Rosenberg \& Sellier

\section{Edizione cartacea}

Data di pubblicazione: 1 dicembre 2016

Paginazione: $563-564$

ISSN: 0039-2944

Notizia bibliografica digitale

Stefano Genetti, «Samuel Beckett, Les Années Godot. Lettres // 1947-1956 », Studi Francesi [Online], 180 (LX | III) | 2016, online dal 01 janvier 2017, consultato il 18 septembre 2020. URL : http://

journals.openedition.org/studifrancesi/5427 ; DOI : https://doi.org/10.4000/studifrancesi.5427

\section{Questo documento è stato generato automaticamente il 18 settembre 2020.}

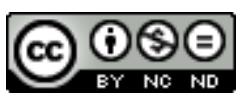

Studi Francesi è distribuita con Licenza Creative Commons Attribuzione - Non commerciale - Non opere derivate 4.0 Internazionale. 


\title{
Samuel Beckett, Les Années Godot. Lettres II 1941-1956
}

\author{
Stefano Genetti
}

\section{NOTIZIA}

SAMUEL BECKETT, Les Années Godot. Lettres II 1941-1956, édition établie par George Craig, Martha Dow Fehsenfeld, Dan Gunn et Lois Overbeck, traduit de l'anglais par André Tapia, Paris, Gallimard, 2015, 761 pp.

1 È un'impresa di inestimabile valore per i lettori, gli spettatori e gli studiosi dell'opera di Beckett la pubblicazione della sua corrispondenza, suddivisa per annate, ciascuna preceduta da una cronologia e ogni volume corredato di un sintetico e documentato apparato di note, di fotografie e profili biografici dei corrispondenti, nonché di un accurato indice dei nomi, titoli e luoghi. Un'impresa ardua - l'elenco delle fonti occupa undici pagine fitte -, costellata di aneddoti curiosi e istruttivi, alcuni dei quali riportati nell' «Introduction générale»; un'impresa prolungatasi per così tanti anni che - si legge in un sorridente inciso - «les éditeurs préfèrent ne pas se souvenir de leur nombre» (p. 24). Mentre, in inglese, è prevista per ottobre 2016 la pubblicazione del quarto e ultimo volume (1966-1989) dell'edizione Cambridge University Press, nell'ottobre 2015 è uscito in Francia il secondo, relativo agli anni del dopoguerra (non figurano infatti lettere risalenti al periodo dell'Occupazione, avendo l'autore disposto che venissero omesse quelle private non attinenti alla sua opera, pari al sessanta per cento per quanto riguarda questo tomo), fino al 1956, quando Beckett scrive per la BBC il radiodramma All That Fall (Tous ceux qui tombent), immagina per il danzatore Deryk Mendel il primo Acte sans paroles e inizia ad assistere alle prove di Fin de partie con Roger Blin et Jean Martin.

2 Segnato dalla guerra, nell'ottobre del 1944, Beckett lascia Roussillon e rientra con la moglie Suzanne - sarà lei a firmare alcune lettere, qui riprodotte, di negoziazione con le case editrici - nell'appartamento di rue des Favorites: è lì, e più tardi nell'isolamento 
della casa di Ussy-sur-Marne, che vengono scritte, sempre più spesso in francese, molte delle pagine indirizzate ad amici vecchi e nuovi, a colleghi, editori, registi, critici e traduttori, compreso il collaboratore di Einaudi Gian Renzo Morteo che, il 17 febbraio 1955, lo contatta in vista di una traduzione, mai apparsa, di Aspettando Godot (pp. 465-466). Sono, dapprima, gli anni del mitico "assedio" creativo, i più produttivi: non solo Godot, cui l'autore deve una notorietà al contempo ambita e che quasi lo infastidisce, ma anche la trilogia composta dai romanzi Molloy, Malone meurt e L'Innommable. Sono, poi, gli anni della crisi creativa inscritta in quel capolavoro misconosciuto che sono i Textes pour Rien, «courts textes avortés» (a George Reavey, 12 maggio 1953, p. 384) cui l'autore finisce per affezionarsi: anni di crisi e di svolta. Soprattutto, sono gli anni in cui egli opta per la scrittura in francese, alla ricerca di quell'indigenza espressiva - «le besoin d'être mal armé» (a Hans Nauman, 17 febbraio 1954, p. 462) - in cui si traduce la poetica della necessità e dell'impossibilità concomitanti esposta nei dialoghi con il saggista e critico d'arte Georges Duthuit. Con quest'ultimo, lo scambio epistolare è tanto più interessante in quanto, come sottolinea Dan GUNN alla fine dell'«Introduction au volume II» (pp.53-90), di quella estetica dell'impedimento traspaiono le profonde implicazioni esistenziali: scrivere è «l'acte sans espoir, calme de sa damnation» (9 giugno 1949, p. 232) di cui Beckett scrive, atto esente da ogni speranza, ma pur sempre in atto.

Non solo la corrispondenza si conferma miniera inesauribile di informazioni sulla gestazione e la diffusione delle opere; non solo fornisce ulteriori prove della meticolosità dell'autore - insiste ad esempio sulla funzione ritmica della ripetizione per esteso dei nomi dei personaggi nel metatesto dell'edizione americana di Waiting for Godot (a Barney e Loly Rosset, 14 dicembre 1953, p. 432) - mitigando in parte la sua presunta ostilità contro ogni forma di adattamento o di trasposizione; non solo abbonda di allusioni, letterarie e non, rivelatrici dei suoi gusti, ad esempio quando esprime la sua ammirazione per i Lieder di Schubert nell'interpretazione di Dieter Fischer-Dieskau - la Winterreise ascoltata alla Salle Gaveau (a Robert Pinget, 8 marzo 1956, p. 593) -, o quando si rammarica di non poter autorizzare un allestimento newyorkese di Godot con Marlon Brando e Buster Keaton, il futuro interprete del suo Film che era andato ad applaudire al Cirque Médrano «avec Lucky», ossia con l'attore Jean Martin (a Pamela Mitchell, 12 gennaio 1954, p. 446 e 17 febbraio 1955, p. 516). La scrittura epistolare si rivela inoltre un laboratorio dove le varie opere vengono commentate, tra premonizioni e risonanze, talvolta anche nel dettaglio. È così che, forte dell'autorità di Rimbaud, Beckett difende l'utilizzo dell'avverbio malinement in una delle numerose lettere a Mania Péron, vedova dell'amico Alfred, deportato nel 1945, e "occhio di lince" che rivede il suo francese (ottobre 1955, p. 547). Come osserva ancora Dan Gunn, il tenore delle missive si fa via via meno polemico e risentito - ma si veda ad esempio il sarcasmo con cui Beckett reagisce alla censura subita dal testo di Godot sui palcoscenici inglesi (a Cyril Lucas, 22 novembre 1955, p. 561). Molto spesso, stralci degli scritti di Beckett sembrano riecheggiare nelle lettere. «Je suis horriblement fatigué et abruti, mais pas encore suffisamment fatigué et abruti», confessa a Rosset, suo editore americano. «Écrire est impossible, mais pas encore suffisamment impossible. Voilà comment je m'emmène en bateau en ce moment» (11 febbraio 1954, p. 457). Se, nella già citata risposta al traduttore tedesco di letteratura irlandese Hans Nauman, Beckett se la cava con "Je ne demande qu'à vous aider», «Mais quant à dire qui je suis, d'où je viens et ce que je fais, tout cela dépasse vraiment ma compétence» (p. 461 e p. 463), allorché riprende il motto di Geulinckx Ubi nihil vales, ibi nihil velis, fa capolino la tipica 
ironia beckettiana: «Il s'agit seulement de s'entendre sur le domaine où l'on ne vaut rien. On ne risque guère d'en exagérer l'étendue» (a Duthuit, primavera 1949, p. 222). Quasi a ribadire l'inestricabilità di scritto e vissuto cui si accennava. 